\title{
mDixon ECG-gated 3-dimensional cardiovascular magnetic resonance angiography in patients with congenital cardiovascular disease
}

Soultana Kourtidou ${ }^{*}$ (D), Marty R. Jones ${ }^{5}$, Ryan A. Moore ${ }^{2}$, Justin T. Tretter ${ }^{2}$, Nicholas J. Ollberding ${ }^{4}$, Eric J. Crotty ${ }^{3}$, Mantosh S. Rattan ${ }^{3}$, Robert J. Fleck ${ }^{3}$ and Michael D. Taylor ${ }^{2}$

\begin{abstract}
Background: Cardiovascular magnetic resonance (CMR) angiography (CMRA) is an important non-invasive imaging tool for congenital heart disease (CHD) and aortopathy patients. The conventional 3D balanced steady-state free precession (bSSFP) sequence is often confounded by imaging artifacts. We sought to compare the respiratory navigated and electrocardiogram (ECG) gated modified Dixon (mDixon) CMRA sequence to conventional nongated dynamic multi-phase contrast enhanced CMRA (CE-CMRA) and bSSFP across a variety of diagnoses.

Methods: We included 24 patients with CHD or aortopathy with CMR performed between September 2017 to December 2017. Each patient had undergone CE-CMRA, followed by a bSSFP and mDixon angiogram. Patients with CMR-incompatible implants or contraindications to contrast were excluded. The studies were rated according to image quality at a scale from 1 (poor) to 4 (excellent) based on diagnostic adequacy, artifact burden, vascular border delineation, myocardium-blood pool contrast, and visualization of pulmonary and systemic veins and coronaries. Contrast-to-noise ratio (CNR), signal-to-noise ratio (SNR) and quantitative vascular measurements were compared between the two gated sequences. Bland-Altman plots were generated to compare paired measures.
\end{abstract}

Results: All scans were diagnostically adequate. Mean (SD) quality scores were $3.4(0.7)$ for the mDixon, $3.2(0.5)$ for the bSSFP and 3.4 (0.5) for the CE-CMRA. Qualitatively, the intracardiac anatomy and myocardium-blood pool definition were better in the bSSFP; however, mDixon images showed enhanced vessel wall sharpness with less blurring surrounding the anatomical borders distally. Coronary origins were identified in all cases. Pulmonary veins were visualized in $92 \%$ of mDixon sequences, $75 \%$ of bSSFP and 96\% of CE-CMRA. Similarly, neck veins were identified in 92, 83 and 96\% respectively. Artifacts prevented vascular measurement in 6/192 (3\%) and 4/192 (2\%) of total vascular measurements for the mDixon and bSSFP, respectively. However, the size of signal void and field distortion were significantly worse in the latter, particularly for flow and metal induced artifacts.

Conclusion: In patients with congenital heart disease, ECG gated mDixon angiography yields high fidelity vascular images including better delineation of head and neck vasculature and pulmonary veins and fewer artifacts than the comparable bSSFP sequence. It should be considered as the preferred strategy for successful CHD imaging in patients with valve stenosis, vascular stents, or metallic implants.

Keywords: Cardiovascular Magnetic Resonance (CMR), Contrast Enhanced Magnetic Resonance Angiography (CE-MRA), Congenital heart disease (CHD), Aortopathy, balanced Steady-State-Free Precession (bSSFP), modified Dixon (mDixon)

\footnotetext{
* Correspondence: sok9030@med.cornell.edu

${ }^{1}$ Weil Cornell Medicine, Department of Pediatrics, Pediatric Cardiology, 525

East 68th St, F-677, New York, NY 10065, USA

Full list of author information is available at the end of the article
}

(c) The Author(s). 2019 Open Access This article is distributed under the terms of the Creative Commons Attribution 4.0 International License (http://creativecommons.org/licenses/by/4.0/), which permits unrestricted use, distribution, and reproduction in any medium, provided you give appropriate credit to the original author(s) and the source, provide a link to the Creative Commons license, and indicate if changes were made. The Creative Commons Public Domain Dedication waiver (http://creativecommons.org/publicdomain/zero/1.0/) applies to the data made available in this article, unless otherwise stated. 


\section{Background}

Cardiovascular magnetic resonance (CMR) angiography (CMRA) is a standard modality used in the routine evaluation of pediatric and adult patients with congenital heart disease (CHD) or aortopathy [1-5]. Conventional dynamic contrast enhanced (CE)-CMRA provides highfidelity three-dimensional (3D) visualization of the heart and chest vasculature without exposure to ionizing radiation or invasive catheterization [6].

Dynamic CE-CMRA is a well-established extensively validated technique proven to be accurate in CHD [3-9]. However, the need for cardiac gated images promoted the development of contemporary electrocardiogram (ECG)-triggered and respiratory gated pulse sequences $[10,11]$. The $3 \mathrm{D}$ balanced steady state free precession (bSSFP) CMRA, often termed "whole-heart" imaging, is an ECG-gated and respiratory-navigated sequence with high signal to noise ratio (SNR) for delineating both intracardiac and extracardiac anatomy [12]. Since the bSSFP contrast is a function of the T2/T1 ratio between tissues, fat appears bright and magnetization preparation schemes are necessary to achieve adequate fat suppression [12]. 3D bSSFP is currently the preferable sequence for coronary artery visualization in CHD [13-15]. Its main disadvantage is sensitivity to flow and susceptibility artifacts [16]. This is can be important to CHD patients who often have both valvar and vessel abnormalities or implanted ferromagnetic devices.

Unlike bSSFP, fat-water separation Dixon-based methods include the underlying $\mathrm{B}_{0}$ distribution in the signal model; therefore image acquisition is less susceptible to field inhomogeneity [16-19]. Modified Dixon (mDixon) CMRA has excellent image quality at $1.5 \mathrm{~T}$ and improve vessel-lumen to fat contrast [20], assist in identifying intramyocardial fatty infiltration in arrhythmogenic right ventricular cardiomyopathy [21, 22], quantify pericardial and epicardial fat in patients with high-risk cardiovascular disease profile [23], and help in the characterization of peripheral arterial occlusive disease [24, 25]. mDixon strategies to reduce artifacts introduced by metal-implants have been a primary focus of musculoskeletal MRI following hip-replacement surgery [26].

To date no studies have reported the application of mDixon CMRA to the evaluation of CHD patients who also often have metalic implants. We sought to investigate the utility of this respiratory navigated and ECG gated mDixon CMRA sequence across a variety of CHD diagnoses and aortopathies in a patient population of a wide range of ages and sizes. We hypothesized that the mDixon sequence would provide high quality diagnostic images with fewer artifacts compared to the conventional wholeheart bSSFP approach.

\section{Methods}

We conducted a retrospective study comparing three different CMRA sequences in CHD patients presenting for CMR evaluation at our institution between September 2017 to December 2017. Consecutive patients with CHD or aortopathy, who had CMR studies with all three sequences were included irrespective of age (children and adults) or diagnosis. We excluded patients with non-CMR compatible implants or contraindications to contrast. The study was approved by our Institutional Review Board which waived informed consent.

\section{CMRA protocol}

The CMR studies were performed in a clinical setting using a $1.5 \mathrm{~T}$ whole-body CMR scanner (Ingenia; Philips Healthcare, Best, The Netherlands) using a standard clinical protocol including dynamic CE-CMRA, Respiratory navigator and ECG gated bSSFP and mDixon whole-heart sequences. Safety screening, compatible monitoring equipment, appropriate imaging coil and cardiac anesthesia for infants requiring sedation were applied in accordance with guideline-directed standards. All studies were performed using contrast (Gadoterate meglumine) at a dose of $0.2 \mathrm{ml} / \mathrm{kg}$ administered by power injector or manually at a flow rate of $2-3 \mathrm{ml} / \mathrm{sec}$ depending on patient age and intravenous catheter size. Each patient underwent a non-gated dynamic multi-phase CE-CMRA followed by the bSSFP and the mDixon pulse sequences.

\section{Sequences}

All three CMRA sequences have been previously described in detail [3, 5, 8-10, 15, 17-19, 27, 28]. Conventional, dynamic CE-CMRA was performed without ECG gating. Five dynamic phases were acquired beginning $8-$ $12 \mathrm{~s}$ after contrast injection. The first 2-3 dynamic phases were acquired during an inspiratory breath hold. Dynamic times were 6-10 s in length. The bSSFP and mDixon sequences were acquired using a beam shaped navigator placed over the hemi-diaphragm opposite the heart. Both sequences were ECG-gated to mid-diastole or end-systole for younger patients with higher heart rates. The 3D-bSSFP is a standard balanced gradient echo steady state precession pulse sequence with a T2-preparation pre-pulse and a spectral pre-saturation pulse. The mDixon sequence is an unbalanced fast gradient echo sequence that achieves fat suppression through chemical shift-based water-fat separation with the modified Dixon technique [19]. The typical acquisition parameters for the whole-heart CMRA protocols are summarized on Table 1. Limited patient-specific adjustments were applied as needed to optimize image acquisition and scan time. The shot durations were decreased to $80 \mathrm{~ms}$ for systolic acquisitions. Three- 
Table 1 Reference Acquisition Parameters for Contrast Enhanced Cardiovascular Magnetic Resonance (CE-MRA), 3D whole-heart Balanced State Free Precession (bSSFP) and modified Dixon (mDixon) sequences

\begin{tabular}{|c|c|c|c|}
\hline Parameter & CE-CMRA & bSSFP & mDixon \\
\hline Average Acquisition Time Goal & Dynamic scan time goal $\sim 12 \mathrm{~s}$ & $3: 11 \mathrm{~min}$ & $3: 08 \mathrm{~min}$ \\
\hline TE (ms) & 0.87 & 2 & $\begin{array}{l}\text { 1st image: } 1.8 \\
\text { 2nd image: } 3.4\end{array}$ \\
\hline TR (ms) & 2.2 & 4.5 & 5.2 \\
\hline Flip Angle (degrees) & $30^{\circ}$ & $90^{\circ}$ & $15^{\circ}$ \\
\hline FOV (mm) & $260 \times 260$ & $260 \times 260$ & $260 \times 260$ \\
\hline Acquired Voxel Size & $1.4 \times 1.4 \times 2.8$ & $1.4 \times 1.4 \times 1$ & $1.4 \times 1.4 \times 1$ \\
\hline Recon Voxel $\left(\mathrm{mm}^{3}\right)$ & $0.9 \times 0.9 \times 0.4$ & $0.9 \times 0.9 \times 1$ & $0.9 \times 0.9 \times 1$ \\
\hline Sense & LR: 2.5; AP: 1.0 & LR: 1.5; AP: 1.0 & LR: 2.0; AP: 1.5 \\
\hline Trigger Delay & NA & Mid-Diastole & Mid-Diastole \\
\hline Shot Duration (ms) & NA & 130 & 130 \\
\hline Navigator Window (mm) & Cartesian k-space acquisition window & 7 & 7 \\
\hline Slice number & 90 & 135 & 135 \\
\hline Bandwidth (Hz/pixel units) & 0.165 & 0.35 & 0.3 \\
\hline
\end{tabular}

Abbreviations: mDixon modified-Dixon, bSSFP balanced Steady State Free Precession, CE-CMRA Contrast-Enhanced Cardiovascular Magnetic Resonance Angiography; TE echo time; TR repetition time; NA not applicable; LR Left Right; AP Anterior Posterior

dimensional volume datasets were obtained in the coronal plane covering the chest, the lower neck vasculature and the upper abdomen.

\section{Image analysis}

The primary image analysis was performed using the phase with the densest arterial contrast for the dynamic CE-CMRA and the water images of the mDixon acquisition. The initial analysis required comparing the mDixon in-phase images and water images for both qualitative and quantitative image quality. The water images were chosen for the primary analysis based their significantly higher SNR and contrast to noise ratio (CNR), better image quality, and improved vessel delineation secondary to effective and homogenous fat suppression (Additional file 1: Figure S1, Additional file 2: Figure S2, and Additional file 3: Figure S3). Two readers (MT; SK) reviewed and graded the studies independently according to overall quality (1-poor, 2-diagnostically adequate, 3 -good, 4-excellent). The scoring parameters were: diagnostic adequacy, presence and effect of artifacts, quality of anatomical borders (blurred versus sharp), myocardium-blood pool contrast, ability to visualize the pulmonary veins, neck veins and coronaries. CNR and SNR were compared in the arterial blood pool and the myocardium, using the equivalent noise in the lungs for reference [20]. Considering the importance of obtaining precise vascular measurements in the CHD population, we performed cross-sectional vascular lumen measurements for the aortic root, ascending aorta, main pulmonary artery (MPA) (or the pulmonary conduit when indicated), left and right branch pulmonary arteries (LPA, RPA), aorta at the level of isthmus, right superior vena cava (R-SVC) and the left anterior descending coronary artery (LAD). Vascular measurements were performed on multiplanar reformatted images in accordance with the 2015 recommendations of the Society for Cardiovascular Magnetic Resonance (SCMR) for obtaining CMR values in adults and children [29]. The measurement location remained consistent among the three sequences independent of image quality. In patients evaluated for known vessel abnormalities, such as stenosis or dilation, the measurement was obtained from the respective area of interest. Quality scores for dynamic, non-gated CE-CMRA were assigned based on the best potential acquisition accounting for the limited intracardiac detail or coronary imaging. The total scan time was recorded for each sequence. Given the fundamental differences in acquisition time and contrast between breath-holding versus respiratory navigator gating techniques, CE-CMRA measurements were not included in the SNR, CNR, vascular and scanning time analyses.

\section{Statistical analysis}

Categorical data were summarized using frequencies and percentages. Continuous values were expressed as mean and standard deviation (SD). Bland-Altman limits of agreement were used to assess the extent of agreement between bSSFP, mDixon and CE-CMRA methodologies. Means (SD), bias (95\% CI), upper and lower limits of agreement for the vascular measures, image quality values and scanning time were calculated. Bland-Altman 
plots were generated to yield a visual comparison of the difference of paired measures against their averages. For binary outcomes, we examined the absolute number and proportion of observations agreeing. Raw-agreement and the weighted kappa statistic were computed to assess inter-rater agreement in the image quality scores. Finally, paired t-tests were applied to test differences in scan times between bSSFP and mDixon. Statistical significance was defined as a $P$-value of $<0.05$. Analyses were conducted using $\mathrm{R}$ v3.4.0 and the tidyverse (v1.2.1), blandr (0.4.3), psych (v1.7.5), and cowplot (v0.9.2) packages.

\section{Results}

Twenty-four consecutive qualifying CMR scans were included from September 2017 - December 2017. One patient was excluded due to disproportionately poor quality and inability to demonstrate any anatomic structures in the bSSFP sequence. Table 2 summarizes the baseline subject characteristics and CHD or aortopathy diagnoses. Ages ranged from 4 months to 40 years, with $50 \%$ of the patients $\leq 20$ years. Fifteen out of the 24 participants were males. Three cases required cardiac anesthesia: a 4month-old, a 4-year-old and a 19-year-old patient with Trisomy 21.

\section{Image quality}

The overall image quality of the mDixon water images was significantly better than the in-phase images. Consequently, the remainder of the results refers to the mDixon water images. The image quality of the mDixon images was comparable, and for certain features, superior to that of the bSSFP. All scans produced clinically diagnostic images with the majority of them rated as "good" or "excellent". No water fat swaps were visible in the mDixon images. There were no anatomic discrepancies with the

Table 2 Baseline Characteristics and Congenital Heart Disease diagnosis

\begin{tabular}{|c|c|c|c|}
\hline Patient & Gender & Age & Diagnosis \\
\hline$\# 1$ & Female & $4 \mathrm{mo}$ & Scimitar syndrome, PAPVC, RPA branch supplying the left lower lung, ASD, VSD, dysplastic pulmonary valve \\
\hline \#2 & Male & 4 yrs & $\begin{array}{l}\text { Heterotaxy, Right atrial isomerism, Complete AVSD, DORV, D-TGA, TAPVC, b/I SVC, s/ p TAPVC repair, b/l Glenn } \\
\text { and pulmonary artery band }\end{array}$ \\
\hline \#3 & Male & $9 y$ rs & Loeys-Dietz Syndrome, s/p valve sparing aortic root replacement with coronary arteries re-implantation \\
\hline \#4 & Female & 13 yrs & TOF s/p VSD closure and transannular patch repair of the right ventricular outflow tract \\
\hline \#5 & Female & 13 yrs & TOF, AVSD, s/p patch repair, pulmonary valve regurgitation, b/l AW regurgitation, ascending aorta dilation \\
\hline \#6 & Female & $14 \mathrm{yrs}$ & $\begin{array}{l}\text { TAPVC, Shone complex, hypoplastic MV, BAV, Coarctation of the aorta, s/p TAPVC repair, s/p coarctation repair } \\
\text { with end to end anastomosis }\end{array}$ \\
\hline \#7 & Female & $14 \mathrm{yrs}$ & TAPVC s/p repair with residual PAPVC \\
\hline$\# 8$ & Male & $16 \mathrm{yrs}$ & Hypoplastic Left Heart Syndrome, s/p Fontan procedure \\
\hline \#9 & Female & 16 yrs & DORV, subaortic VSD s/p patch VSD closure; s/p resection of subaortic membrane and RV muscle bundle \\
\hline \#10 & Male & 17 yrs & Congenital LPA hypoplasia \\
\hline \#11 & Female & 19 yrs & Pulmonary valve stenosis s/p balloon valvuloplasty \\
\hline \#12 & Male & 19 yrs & Down syndrome, Unrepaired Coarctation of aorta, congenital stenoses of the branch pulmonary arteries \\
\hline$\# 13$ & Male & 20 yrs & TOF s/p VSD and transannular patch repair, mechanical pulmonary valve replacement, aortic root dilation \\
\hline \#14 & Male & 24 yrs & D-TGA s/p arterial switch; RPA narrowing, hypoplastic LPA, dilated aortic root, aortic valve regurgitation \\
\hline \#15 & Male & 24 yrs & TOF s/p complete repair, aortic root dilation, high LCA origin, pectus excavatum \\
\hline \#16 & Male & 27 yrs & Pulmonary vale atresia, VSD, discontinuous branch pulmonary arteries s/p repair \\
\hline \#17 & Male & 27 yrs & BAV, dilated aortic root \\
\hline \#18 & Male & 27 yrs & VSD, Coarctation of aorta s/p SCA flap repair, distal TAA pseudoaneurysm, dilated aortic root \\
\hline \#19 & Female & 27 yrs & Unrepaired PAPVC, BAV \\
\hline \#20 & Male & 30 yrs & BAV s/p repair with residual stenosis, ascending aorta dilation \\
\hline \#21 & Male & 31 yrs & BAV s/p bioprosthetic valve placement \\
\hline \#22 & Male & 38 yrs & TOF s/p RV-PA conduit replacement, with residual conduit stenosis and aortic root dilation \\
\hline \#23 & Male & 40 yrs & BAV, s/p mechanical valve placement, aortic root and ascending aorta dilation \\
\hline \#24 & Female & 40 yrs & Ehlers-Danlos syndrome s/p aortic root replacement; MRI-compatible Pacemaker \\
\hline
\end{tabular}

Abbreviations: mo: months, yrs: years, PAPVC Partial Anomalous Pulmonary Venous Connection, RPA Right Pulmonary Artery, ASD Atrial Septal Defect, VSD Ventricular Septal Defect, AVSD Atrio-Ventricular Septal Defect, DORV Double Outlet Right Ventricle, D-TGA D-Transposition of the Great Arteries, TAPVC Total Anomalous Pulmonary Venous Connection, B/L Bilateral, SVC Superior Vena Cava, S/P Status Post, TOF Tetralogy of Fallot, AVV Atrioventricular Valve, MV Mitral Valve, BAV Bicuspid Aortic Valve, LPA Left Pulmonary Artery, SCA Subclavian Artery, TAA Transverse Aortic Arch, RV-PA Right Ventricle to Pulmonary Artery 

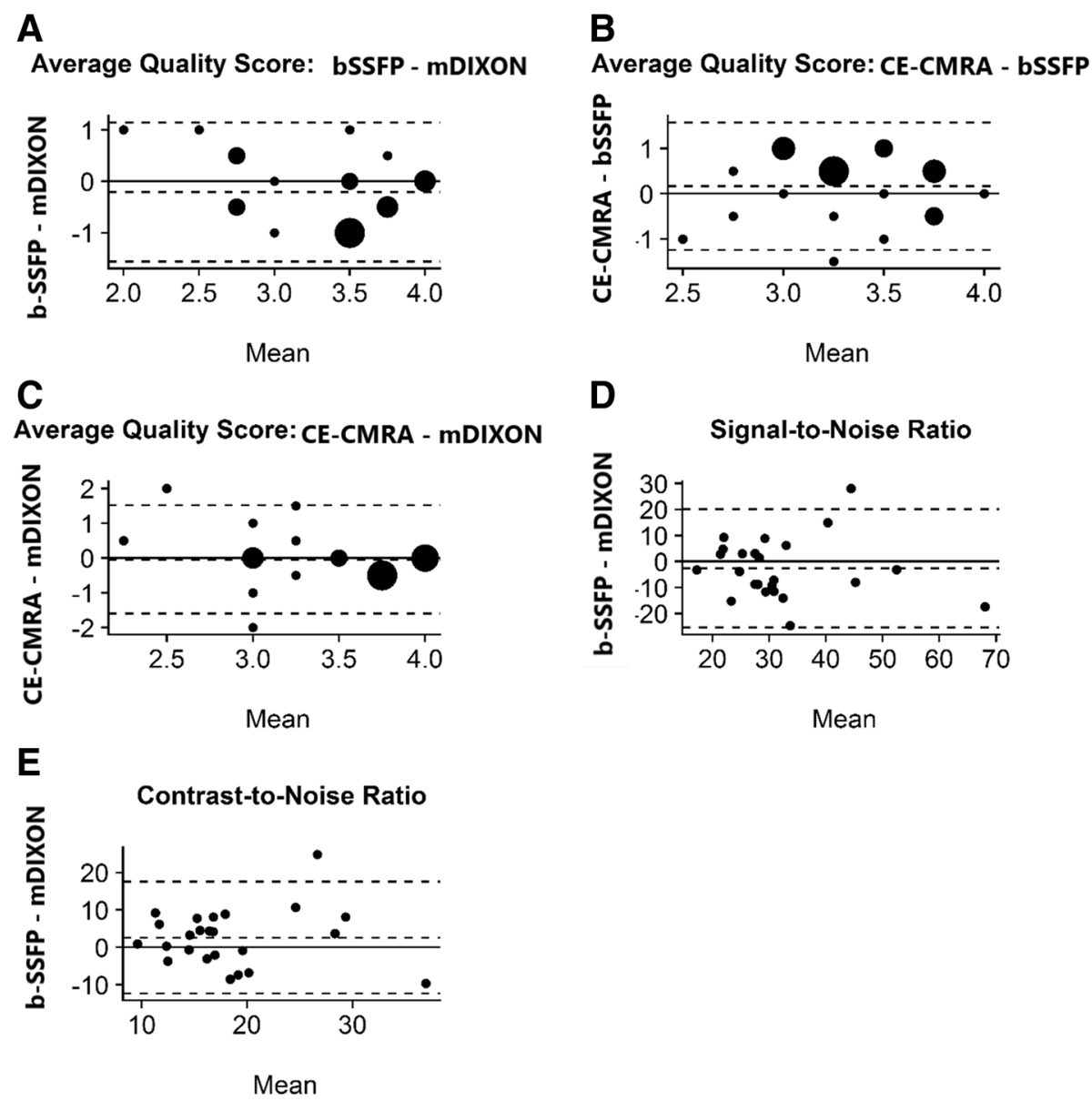

Fig. 1 Bland-Altman Plots for Image Quality Scores between Balanced Steady State Free Precession (bSSFP)-modified Dixon (mDixon) (a), Contrast Enhanced (CE)-Cardiovascular Magnetic Resonance Angiography (CMRA)-bSSFP (b), CE-CMRA-mDixon (c); Signal-to-Noise Ratio (SNR) (d) and Contrast-to-Noise Ratio (CNR) (e) between bSSFP-mDixon. The $y$-axis provides the difference of the paired measures. The $x$-axis provides the mean of the paired measures. The dotted lines provide the mean difference and the $95 \%$ limits of agreement

reported study findings. The Bland-Altman plots revealed acceptable agreement between the bSSFP-mDixon, CECMRA-bSSFP and CE-CMRA-mDixon pairs with respect to their average image quality scores (Fig. 1, a-c). The mean quality values, averaged for the two reviewers, were 3.4 (0.7) for the mDixon, $3.2(0.5)$ for the bSSFP and 3.4 (0.5) for the CE-CMRA. The bias (mean of the observed differences) between mDixon and bSSFP was - 0.21(95\% CI: $-0.50 ; 0.08)$ and the limits of agreement (LOA) and 95\% CIs were $-1.56(-2.07 ;-1.06)$ and 1.14 (95\% CI: $0.64 ; 1.65)$ for the lower and upper LOAs, respectively. The bias (mean of the observed differences) between mDixon and CE-CMRA was 0.04 (95\% CI: - 0.29; 0.38) and the LOA $(95 \% \mathrm{CI})$ were $-1.51(-2.09 ;-0.93)$ and 1.6 (1.02; 2.18) for the lower and upper LOAs, respectively. The weighted kappa statistic demonstrated variable interobserver agreement between the two reviewers (MT, SK) depending on the sequence type. Specifically, it was good for the mDixon [raw:71\%; weighted kappa $=0.62(0.41$,
0.83)], moderate for the bSSFP [raw: 63\%; weighted kappa $=0.46(0.22,0.70)]$ and fair for the CE-CMRA [raw: $46 \%$; weighted kappa $=0.37(0.03,0.70)]$.

With regards to individual image quality elements, mDixon depicted the entire field of view better as compared to CE-CMRA and bSSFP, offering a comprehensive assessment of the neck vasculature, the chest cavity and the upper abdomen anatomy. Qualitatively, the intracardiac anatomy and myocardium-blood pool definition were better in the bSSFP; however, mDixon images showed superior vessel wall sharpness with less blurring in distal vessels, particularly in vessels near the edges of the field of view and vessels within the lung fields, (Fig. 2) . In agreement with the above, pulmonary veins were visualized in $92 \%$ of the mDixon sequences, while the respective percentages were $75 \%$ for bSSFP and $96 \%$ for CE-CMRA, (Fig. 3). Adequate visualization of the neck veins was achieved in $92 \%$ of mDixon, $83 \%$ of bSSFP and $96 \%$ of CE-CMRA images, (Figs. 2, 3, 4, 5 and 6). 


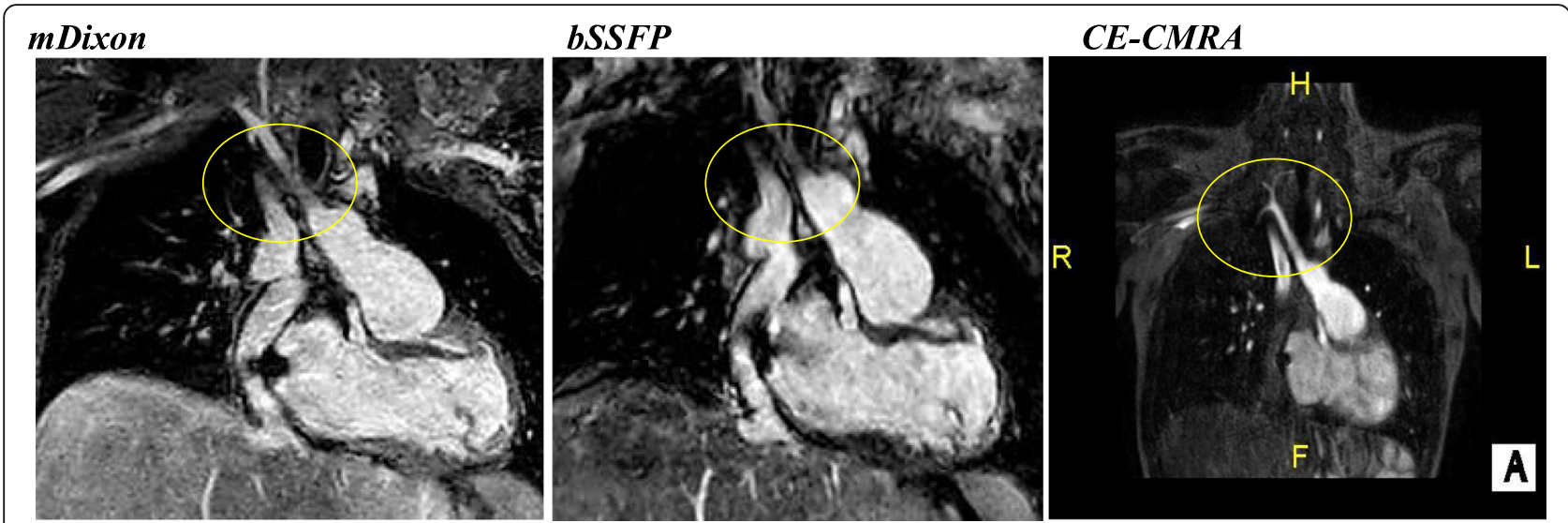

Fig. 2 16-year-old with hypoplastic left heart syndrome s/p Fontan palliation. Demonstration of complex CHD anatomy by whole heart sequences and standard CE-CMRA. Smaller flow artifact in the Fontan fenestration, lung and neck vasculature, comparable quality of myocardium-blood boarder, sharper diaphragm/liver/heart, chest cavity boarders. Better SVC Fontan anastomosis seen in balanced SSFP. LesS detailed visualization of the anatomy in the CE-CMRA

Notably, we were able to identify arterio-venous collateral vessels within the lungs, as well as, aortopulmonary collateral vessels in both mDixon and bSSFP images; yet, their distal courses were better displayed in the mDixon images. Both whole-heart sequences performed very well in demonstrating the coronary artery origin and distal courses, (Fig. 4). Thus, contrast intensity and incomplete fat suppression artifact subjectively interfered with quality and in 3 cases it did not allow visualization of LAD at its full length. Despite our observations, the Bland-Altman plots did not display any obvious differences for the SNR and CNR magnitude between bSSFP and mDixon, (Fig. 1, d-e). In Figs. 2, 4, and 5, the diaphragm is significantly easier to see in the mDixon images. This is likely secondary to B0 inhomogenities as the
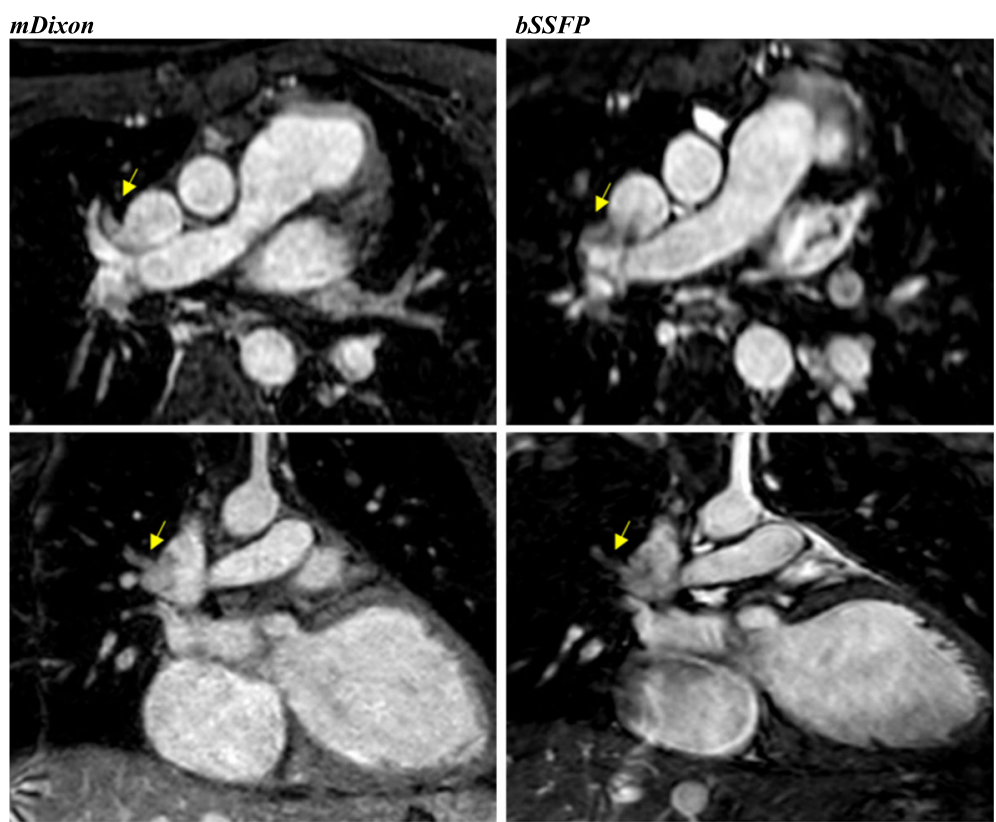

Fig. 3 15-year-old girl with history of total anomalous pulmonary venous connection (TAPVC) s/p repair with residual partial anomalous pulmonary venous connection (PAPVC); the right upper pulmonary vein drains into the SVC. The abnormal course and connection (arrow) is only partially visualized in the bSSFP 

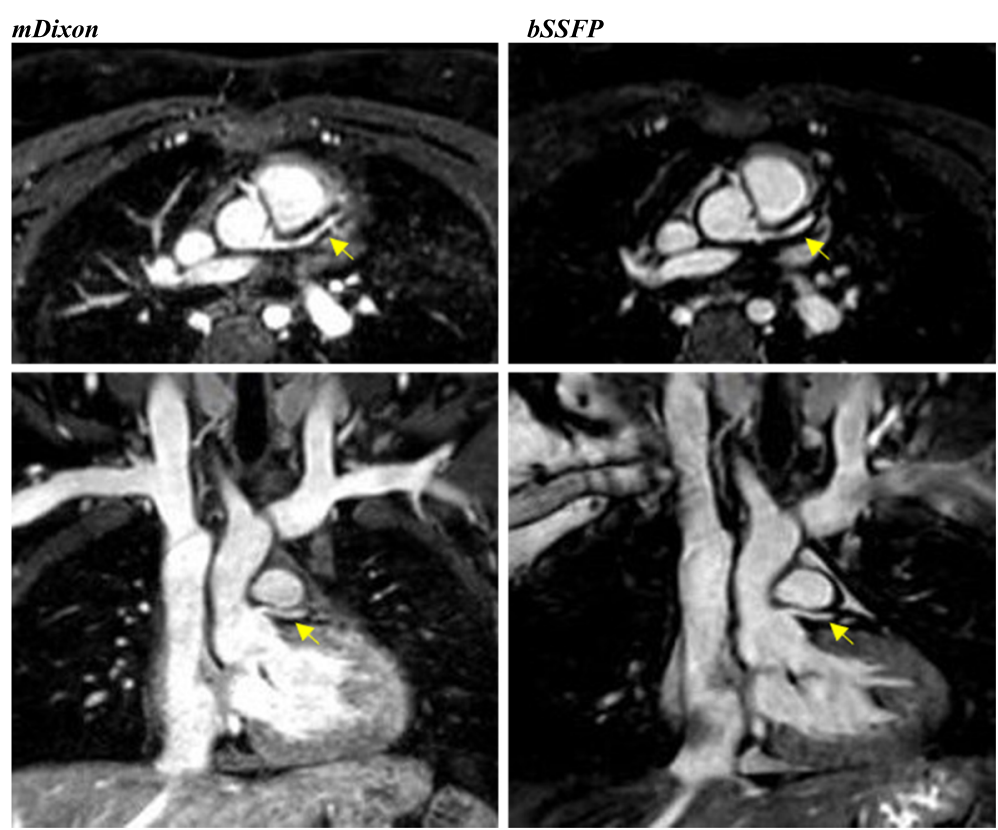

Fig. 4 19-year-old female with unrepaired coarctation of the aorta. Comparable performance of the whole-heart sequences in demonstrating the origins and proximal coronary artery courses. Also, note the improved visualization of the neck and lung vasculature as well as the right hemidiaphragm in the mDixon image

respiratory navigator respiratory gating was identical for both sequences.

\section{Artifacts}

Of the 24 cases, 17 had some type of artifact in one or more sequences. Given the anatomic complexity and the high likelihood of prior surgical or catheter induced intervention, susceptibility and flow-related artifacts were the most frequently encountered types. Such artifact burden was notably less appreciable in mDixon versus bSSFP and CE-CMRA. The predominant artifacts in the bSSFP images were B0 inhomogeneities and flow artifacts; in the mDixon images they were B0 inhomogeneities and incomplete fat/water separation. Figures 5 and 6 show the impact of an artificial aortic valve and an CMRcompatible pacemaker, respectively, among the three corresponding techniques. Motion blurring was typically associated with the CE-CMRA images. mDixon demonstrated successful fat-suppression resulting to minimal chemical-shift artifact around the pericardial and vessel wall borders, including the coronary arteries. The presence of artifact prevented us from performing designated vascular measurements in 6/192 (3\%) of cases in the mDixon and 4/192 (2\%) in the bSSFP images, however, the size of the obscured area was significantly worse in the latter sequence. For the

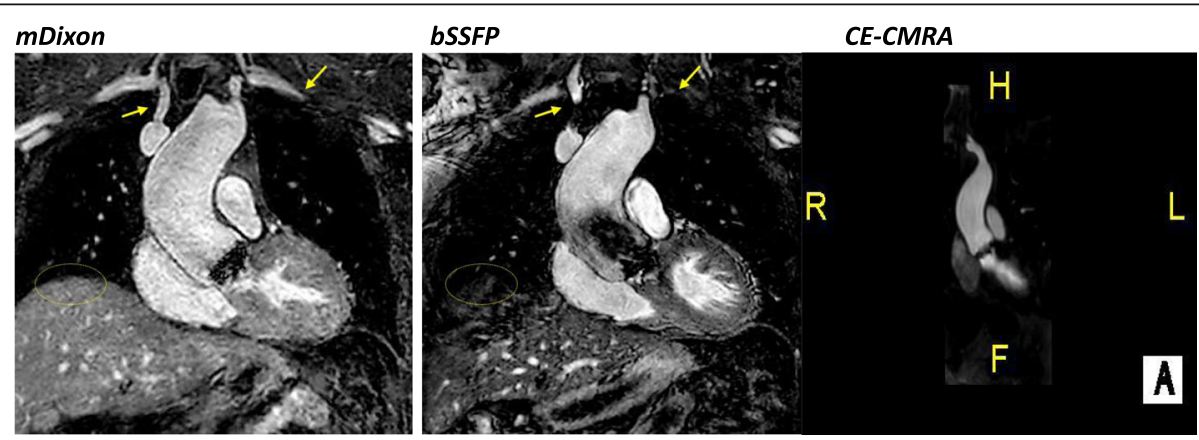

Fig. 5 40-year-old male with history of BAV s/p mechanical valve (St. Jude) placement with aortic root and ascending aorta dilation. The flow artifact is significantly reduced in the mDixon image. The yellow arrows denote veins obscured by artifacts in the balanced SSFP image. Also, note the improved visualization of the right hemi-diaphragm in the mDixon image (Elliptical ROI). CE-CMRA phase focused in the aortic root and ascending aorta; small flow artifact 

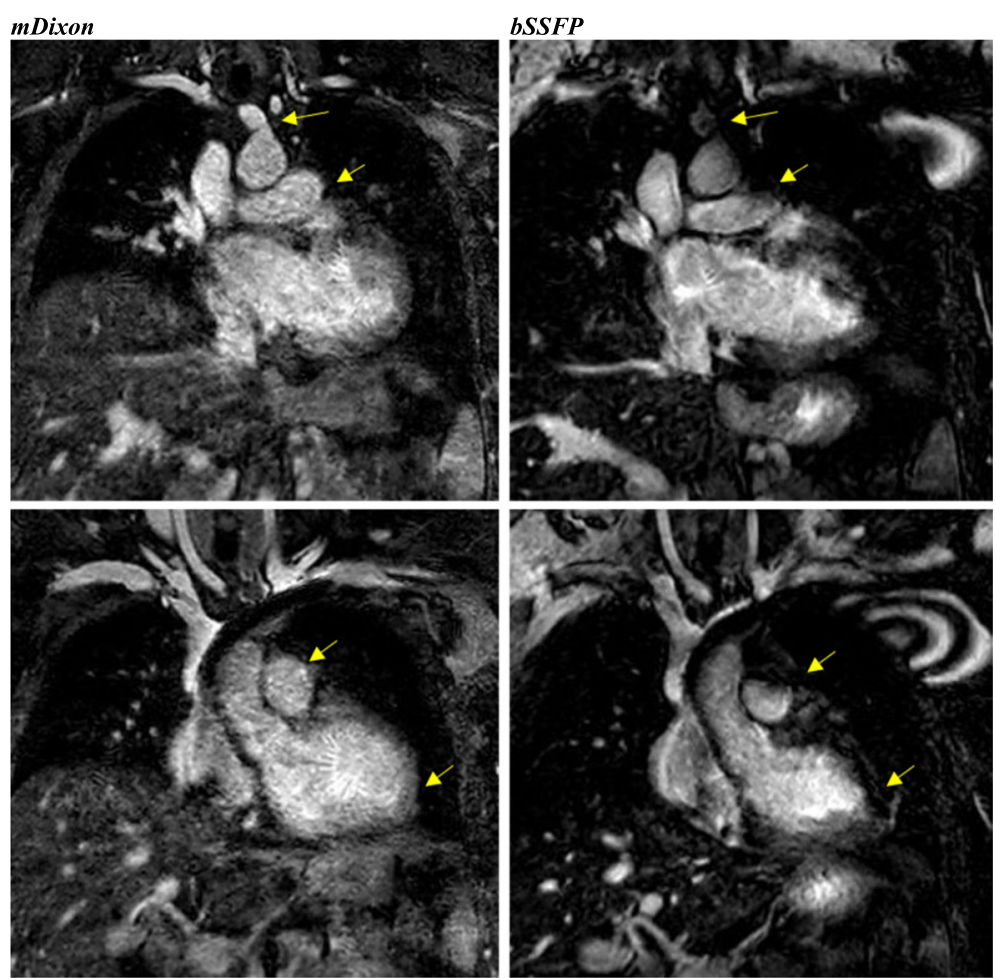

Fig. 6 40-year-old with Ehlers-Danlos syndrome and left ventricular non-compaction, s/p CMR-compatible pacemaker. The image quality in both is degraded. The main pulmonary artery (MPA) and head and neck vasculature are better visualized in the mDixon image. However, the endomyocardial border is better defined in the bSSFP image, a common finding in the study

mDixon, of the 6 inadequately visualized vascular specific locations, two were obscured by metal artifact, one case was related to flow-artifact in the main pulmonary artery and 3 cases were attributed to limited visualization of coronary arteries secondary to the high contrast intensity or incomplete fat suppression. In contrast, susceptibility was the only type of artifact that limited our ability to perform vascular measurements in bSSFP.

A summary table with detailed Bland-Altman agreement estimates for all the assessed image quality parameters (Image quality score, SNR, CNR, artifact preventing the performance of vascular measurement, visualization of neck and pulmonary veins) is included in supplemental analysis (Additional file 4: Table S1 and Additional file 5: Table S2).

\section{Temporal order}

Both whole-heart sequences were performed after contrast administration for the CE-CMRA. The mDixon preceded the bSSFP in 15 out of the 24 studies. No impact of the temporal order was detected for the image quality scores, SNR, CNR, presence of artifact preventing a measurement, or visualization of venous structures, (Fig. 7).

\section{Vascular measurements}

We performed a comprehensive assessment of the chest vasculature to include both arterial and venous structures with wide-ranging sizes. Bland-Altman analyses for 8 cross-sectional vascular measurements are summarized in Table 3. The bias and 95\% limits of agreement between each set of vascular diameters showed no systematic bias and less than 10\% measurement difference between all mDixon and bSSFP estimates irrespective of the vessel type and size. The analysis was also performed using the mDixon water images. There was no systematic bias in the water and in-phase mDixon image measurements (Additional file 6: Table S3).

\section{Time efficiency}

The respective acquisition duration for the free-breathing, 135-slice whole heart sequences were comparable: 6.6 (2.6) minutes for the bSSFP and 7.4 (3.4) minutes for the mDixon, $(p=0.20)$. The average navigator efficiency ranged between 40 and $60 \%$.

\section{Discussion}

This is the first study validating the mDixon whole-heart CMRA technique in CHD and aortic pathology. High 
A B

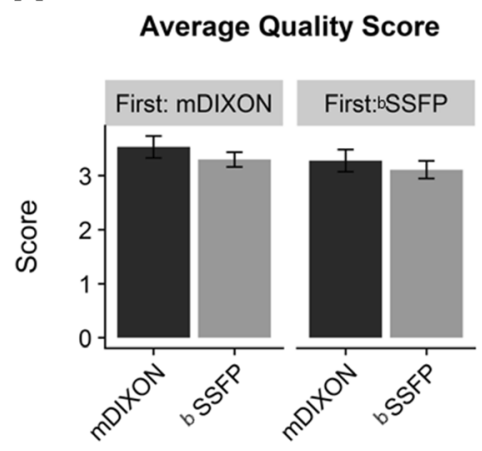

D

Artifact Preventing Vascular Measurement

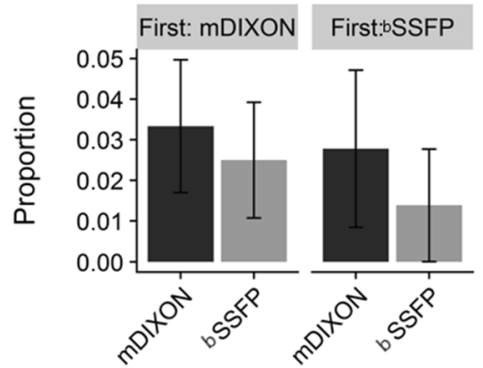

B

Signal-to-Noise Ratio

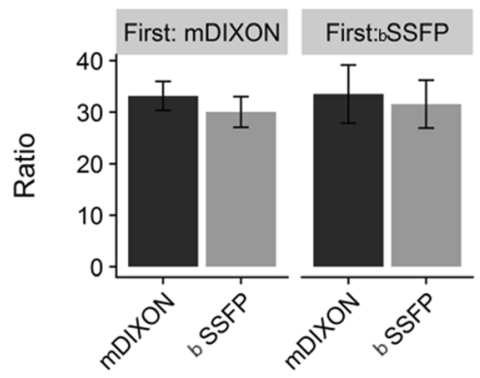

E

Visualization of Neck Veins

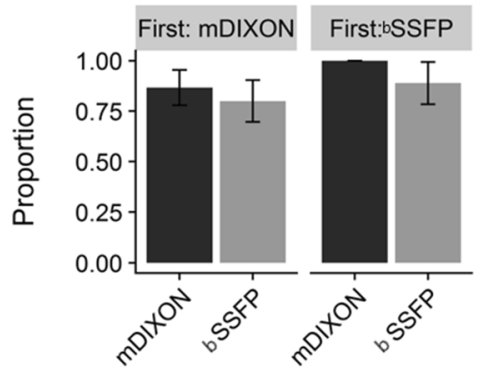

C

\section{Contrast-to-Noise Ratio}

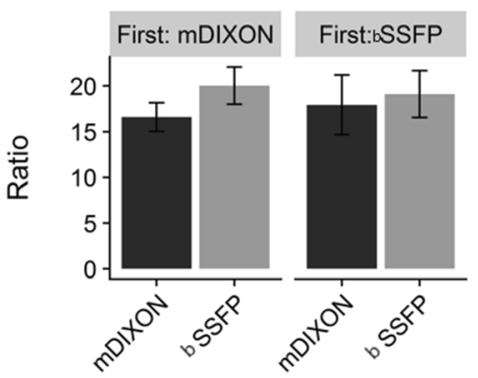

$\mathbf{F}$

Visualization of Pulmonary Veins

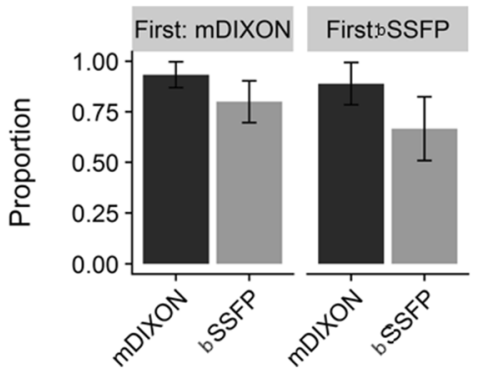

Fig. 7 Effect of temporal order of sequence in image quality parameters: (a) Average quality score, (b) Signal-to-Noise Ratio, (c) Contrast-to-Noise Ratio, (d) Artifact Preventing Vascular Measurement, (e) Visualization of Neck Veins, (f) Visualization of Pulmonary Veins

quality mDixon CMRA images were obtained without the need for cardiac anesthesia, indicating that mDixon could be integrated into the CMR workflow, particularly for patients with expected flow and susceptibility artifact, systemic or pulmonary venous pathology.
Even though our pilot investigation warrants full-scale evaluation, our diverse patient sample supports the applicability of mDixon CMRA in patients of variable age, size and heart rate independent of prior catheter or surgical interventions. A wide spectrum of CHD is represented,

Table 3 Summary of agreement estimates for quantitative vascular measurements

\begin{tabular}{|c|c|c|c|c|c|}
\hline Cross-sectional Vessel Diameter & $\begin{array}{l}\text { mDixon } \\
\text { Mean (SD) }\end{array}$ & $\begin{array}{l}\text { bSSFP } \\
\text { Mean (SD) }\end{array}$ & $\begin{array}{l}\text { Bias } \\
(95 \% \mathrm{Cl})\end{array}$ & $\begin{array}{l}\text { Lower LOA } \\
(95 \% \mathrm{Cl})\end{array}$ & $\begin{array}{l}\text { Upper LOA } \\
(95 \% \mathrm{Cl})\end{array}$ \\
\hline Aortic Root (mm) & $29.4(9.4)$ & $29.6(8.8)$ & $\begin{array}{l}0.09 \\
(-0.58 ; 0.76)\end{array}$ & $\begin{array}{l}-2.87 \\
(-4.03 ;-1.71)\end{array}$ & $\begin{array}{l}3.05 \\
(1.89 ; 4.21)\end{array}$ \\
\hline Ascending Aorta (mm) & $27.0(8.9)$ & $26.0(9.8)$ & $\begin{array}{l}-0.22 \\
(-1.02 ; 0.59)\end{array}$ & $\begin{array}{l}-3.86 \\
(-5.25 ;-2.47)\end{array}$ & $\begin{array}{l}3.42 \\
(2.03 ; 4.82)\end{array}$ \\
\hline Main Pulmonary Artery/Conduit (mm) & $22.6(6.5)$ & $22.0(6.6)$ & $\begin{array}{l}-0.20 \\
(-1.18 ; 0.78)\end{array}$ & $\begin{array}{l}-4.30 \\
(-6.00 ;-2.09)\end{array}$ & $\begin{array}{l}3.9 \\
(2.20 ; 5.60)\end{array}$ \\
\hline Left Pulmonary Artery (mm) & $15.6(4.4)$ & $16.2(5.1)$ & $\begin{array}{l}0.95 \\
(-0.18 ; 2.09)\end{array}$ & $\begin{array}{l}-4.05 \\
(-6.02 ;-2.09)\end{array}$ & $\begin{array}{l}5.96 \\
(3.99 ; 7.93)\end{array}$ \\
\hline Right Pulmonary Artery (mm) & $15.3(4.0)$ & $15.3(4.2)$ & $\begin{array}{l}-0.09 \\
(-0.93 ; 0.76)\end{array}$ & $\begin{array}{l}-3.91 \\
(-5.38 ;-2.45)\end{array}$ & $\begin{array}{l}3.74 \\
(2.27 ; 5.20)\end{array}$ \\
\hline Descending Aorta (isthmus) (mm) & $16.7(4.8)$ & $16.5(4.8)$ & $\begin{array}{l}-0.32 \\
(-0.92 ; 0.28)\end{array}$ & $\begin{array}{l}-2.98 \\
(-4.03 ;-1.94)\end{array}$ & $\begin{array}{l}2.35 \\
(1.30 ; 3.39)\end{array}$ \\
\hline Left Anterior Descending Coronary Artery (mm) & $3.8(0.9)$ & $3.8(0.8)$ & $\begin{array}{l}0.03 \\
(-0.19 ; 0.24)\end{array}$ & $\begin{array}{l}-0.84 \\
(-1.21 ;-0.47)\end{array}$ & $\begin{array}{l}0.90 \\
(0.52 ; 1.27)\end{array}$ \\
\hline Superior Vena Cava (mm) & $16.4(4.1)$ & $15.9(4.7)$ & $\begin{array}{l}-0.48 \\
(-1.00 ; 0.04)\end{array}$ & $\begin{array}{l}-2.83 \\
(-3.73 ;-1.93)\end{array}$ & $\begin{array}{l}1.88 \\
(0.98 ; 2.78)\end{array}$ \\
\hline
\end{tabular}


including complex heterotaxy, different stages of single ventricle palliation, pulmonary venous abnormalities, valvular and great artery disease. All important anatomy was demonstrated with accurate morphologic detail. The datasets revealed consistent findings with regards to key reporting elements and they were deemed reliable for long-term surveillance and interpretation of changes over time for pre-procedural planning $[3,4]$.

In addition to diagnostic adequacy, we assessed a comprehensive range of image quality features and examined the accuracy of quantitative measurements in the coronary and peripheral chest vasculature between mDixon versus the two reference-conventioanl CMRA techniques, namely CE-CMRA and 3D whole-heart balanced bSSFP. In terms of visual quality scores, mDixon displayed adequate tissue characterization, sharper distal vascular anatomic borders and clearer spatial relationships among the extracardiac surrounding chest structures that interfere with fat tissue and air space within the field of view. Overall, the generated set of water images were characterized by robust homogeneous fat suppression, acceptable SNR, CNR [16, 29-31] and significantly less artifact burden. While conventional whole-heart techniques depend primarily on short T1relaxation time and frequency-selective fat suppression, modified Dixon is a chemical shift-encoded fat-water separation methodology [17, 19]. Consequently, it is more resilient to background B0 and B1 magnetic field inhomogeneities that can be particularly strong in the thoracic cavity [20]. Due to ECG and respiratory gating, both whole heart sequences had minimal ghosting artifacts secondary to motion and spatial misregistration, $[16,32]$, though, mDixon demonstratesd significantly less sensitivity to blood flow and chemical shift distortions $[21,34]$. The SNR has a complex dependency on the pulse sequence, parallel imaging factor, timing after contrast, and k-space parameters. The inherent SNR is higher for the bSSFP sequence, but the image noise is also higher. The expectation was that the bSSFP SNR would be higher, but the noise dominated and the SNR for the mDixon was higher.

Therefore, mDixon performance was superior in anatomic regions with disturbed blood flow, including stenotic and regurgitant valves, vascular anastomoses, stenotic or dilated vascular segments. Likewise, mDixon showed less susceptibility artifact induced by ferromagnetic implants as compared to bSSFP and CE-CMRA techniques. We observed smaller signal voids and superior fat suppression in the periprosthetic area in a variety of metal prostheses, from simple sternal wires and vascular plugs to artificial intracardiac valves, vascular stents and MRI compatible pacemakers. Experimental and limited adult publications on breast [34] and skeletal
MRI image quality in the presence of metal prosthesis [26] have shown discrepant results between short tau inversion recovery (STIR) pulse and Dixon methodologies. Our study is the first to offer critical information on whole-heart sequence performance in the chest that is known for being technically challenging and prone to local magnetic field distortion. It is worth mentioning, that "any type of artifact" prevented us from performing a vascular measurement in $6 \mathrm{mDixon}$ versus 4 bSSFP and 6 CE-CMRA images. Thus, metal interference occurred in only 2 out of the $6 \mathrm{mDixon}$ cases as detailed previously.

Visualization of the vessel course and lumen patency are critically important in the CHD population for diagnosis and planning of transcatheter or surgical interventions. Such anatomic structures are challenging to delineate by conventional transthoracic echocardiography and invasive $\mathrm{X}$-ray angiography has historically been the reference standard [35]. Geva et al. demonstrated adequate diagnostic capability of gadolinium-enhanced 3D CMRA in a large cohort of patients with congenital and acquired pulmonary and systemic venous abnormalities who had undergone cardiac catheterization [8]. In our study, the diagnostic yield of mDixon in depicting the pulmonary and neck veins was consistently comparable to that of the CE-CMRA and higher than the bSSFP. Our results are in line with existing adult literature showing high spatial resolution and vessel-to-background contrast in imaging of peripheral arteries via Dixon-based fat-free CMRA [24, 25]. The clinical relevance of applying mDixon sequences consists in providing high quality intracardiac information simultaneously with arterial and venous vascular data, including not only the pulmonary and neck veins, but also collateral vessels that play a pivotal role for targeted therapeutic interventions.

With regards to the coronary artery anatomy, both whole-heart methodologies allowed reliable identification of the origins and proximal courses with comparable fat suppression, contrast and vessel wall sharpness and overall visual quality scores. The visualization of the mid- to distal LAD was inadequate in 3 mDixon images versus none in the bSSFP. Heart rate did not seem to have any effect as the patients belonged to diverse age groups $(4 \mathrm{mo}, 13 \mathrm{yrs}$., 27 yrs., $40 \mathrm{yrs}$ ). Three of those sequences were performed prior to the bSSFP. Additional analysis to further investigate the temporal effect of contrast showed no meaningful difference when mDixon was performed before the bSSFP. In the presence of contrast, both fat and blood demonstrate a significantly bright signal so fat suppression is important in delineating differences between tissues [36]. Currently, the contrast enhanced whole-heart bSSFP is the protocol of choice in pediatric and adult CHD coronary CMRA $[3-5,10,14,15,37]$. In a small pilot study of 8 healthy volunteers, Nezafat et al. demonstrated significantly 
higher CNR and SNR in the coronary artery tree with Dixon water-fat separation than spectral presaturation with inversion recovery (SPIR) fat suppression at $3 \mathrm{~T}$, even without IV contrast administration [33]. mDixon techniques have been proven useful in peripheral CMRA as well [38, 39]. In contrast, Börnert et al., found that SNR and CNR tended to drop in bSSFP sequences after gadolinium administration and suggested that fat-saturated images may be advantageous in demonstrating small epicardial vessel structures and tissue boundaries [20]. Within the same context, we observed that the myocardium-blood pool contrast was significantly better in the bSSFP images due to smoother contrast intensity and somewhat finer delineation of the cardiac tissues. Given the advantageous perivascular fat suppression in combination with the possibility of contrast interference with visual quality, non-contrast mDixon CMRA could be a future consideration.

\section{Limitations}

Our findings are limited by the retrospective study design and the relatively small sample size. Consequently, we refrained from performing certain quantitative statistical comparisons which would have required a larger cohort. The interrater agreement varied profoundly depending on the type of the sequence. It is plausible that the unblinded process could have generated observer bias. Interestingly, the kappa statistic was highest for the mDixon and lowest for the CE-CMRA and therefore, some of the discrepancy may be attributed to the different levels of experience between the reviewers when rating the best possible CECMRA quality images. mDixon works very well in our lab, however, generalizability of our results cannot be concluded. We attempted to maintain scanner settings similar for the two sequences to achieve comparable conditions; further manipulations may have had optimized some quality features. Despite the fact that our whole-heart scanning times are similar, we acknowledge that the mDixon images were acquired with a slightly higher parallel imaging factor that may degrade the image quality. This was done to make the image acquisition times approximately equal between the two sequences. Lastly, there is supporting evidence that cardiac fat carries important diagnostic information in differentiating pericardial or endo-myocardial fat infiltrative conditions [16]. Analysis of the mDixon fatimages was beyond the scope of this preliminary study, however, future studies should further investigate such source of information in the CHD population.

\section{Conclusion}

In our pilot retrospective study, mDixon 3D ECG gated CMRA yielded precise delineation of complex cardiovascular anatomies in freely breathing patients with decreased artifacts compared to our standard bSSFP 3D ECG gated sequence. The mDixon technique performed comparably for evaluation of intracardiac and extracardiac anatomy. Importantly, mDixon suffered less artifact burden and may be an excellent alternative to bSSFP as the preferred strategy for gated angiography of CHD and aortopathy patients in the presence of disturbed blood flow, metallic implants, systemic or pulmonary venous disease.

\section{Additional files}

\begin{abstract}
Additional file 1: Figure S1. Example images from mDixon acquisition The water, fat, in-phase, and opposed-phase images are shown in the panels A-D. Note the improved vessel delineation in the water image (A) secondary to fat signal suppression. (DOCX $230 \mathrm{~kb}$ )
\end{abstract}

Additional file 2: Figure S2. Average quality score for mDixon water and in-phase images. ${ }^{* * *} p$-value $<0.01$. (DOCX $139 \mathrm{~kb}$ )

Additional file 3: Figure S3. Signal to noise and contrast to noise ratios for water and in-phase images. There is a significant difference in both parameters. ${ }^{* * *}$ p-value $<0.001$. (DOCX 187 kb)

Additional file 4: Table S1. Summary of agreement estimates for Image Quality measures: Average Quality Score, SNR and CNR. (DOCX 13 $\mathrm{kb})$

Additional file 5: Table S2. Agreement estimates for qualitative image quality measures. (DOCX $14 \mathrm{~kb}$ )

Additional file 6: Table S3. Quantitative comparison of the mDixon water and in-phase images for vessel measurements. (DOCX $16 \mathrm{~kb}$ )

\begin{abstract}
Abbreviations
3D: Three dimensional; ASD: Atrial Septal Defect; AVSD: Atrio-Ventricular Septal Defect; AW: Atrioventricular Valve; B/L: Bilateral; BAV: Bicuspid Aortic Valve; bSSFP: balanced Steady-State Free Precession;; CE: Contrast Enhanced; CHD: Congenital Heart Disease; Cl: Confidence Interval; CMR: Cardiovascular Magnetic Resonance; CMRA: Cardiovascular Magnetic Resonance Angiography; CNR: Contrast-to-Noise Ratio; DORV: Double Outlet Right Ventricle; D-TGA: D-Transposition of the Great Arteries;

ECG: Electrocardiogram; LAD: Left Anterior Descending coronary artery; LOA: Limit of Agreement; LPA: Left Pulmonary Artery; mDixon: modified Dixon; MPA: Main Pulmonary Artery; MV: Mitral Valve; PAPVC: Partial Anomalous Pulmonary Venous Connection; RPA: Right Pulmonary Artery; RVPA: Right Ventricle to Pulmonary Artery:i S/P: Status Post; SCA: Subclavian Artery; SCMR: Society for Cardiovascular Magnetic Resonance; SD: Standard Deviation; SNR: Signal-to-Noise Ratio; SPIR: Spectral Presaturation with Inversion Recovery; STIR: Short tau Inversion Recovery; SVC: Superior Vena Cava; TAA: Transverse Aortic Arch; TAPVC: Total Anomalous Pulmonary Venous Connection; TOF: Tetralogy of Fallot; VSD: Ventricular Septal Defect
\end{abstract}

\section{Acknowledgements}

We sincerely thank our Cincinnati Children's CMR technologists for their continuous support with our research efforts: C. Bazan, V. Berberich, S. Dearth, A. Lamont, S. Kossen and J. Tiefermann.

\section{Authors' contributions}

MT made substantial contributions to the design and improvement of CMR scanning sequences, the conception and design of the project, the acquisition, analysis, interpretation of data and mentored SK throughout the manuscript preparation. SK contributed to the acquisition and interpretation of data, drafted and finalized the manuscript. MJ made substantial contributions to the design and improvement of CMR scanning sequences. NO performed statistical analysis and critically reviewed manuscript. RM, JT, EC, MR and RF assisted in drafting the work and revising it critically for important intellectual content. All authors provided final approval of the version to be published.

\section{Funding}

This work was supported by the Heart Institute Research Center, Cincinnati Children's Hospital Medical Center, $\mathrm{OH}$. 


\section{Availability of data and materials}

The dataset generated and/or analyzed during the current study are available from the corresponding author on reasonable request.

\section{Ethics approval and consent to participate}

The study protocol was approved by the Institutional Board Review of Cincinnati Children's Hospital Medical Center, OH. Written informed consent was obtained from the participants for publication of their individual details and images in this manuscript. The consent forms are held at the authors' institution and are available for review by the Editor-in-Chief.

\section{Consent for publication}

All the subjects provided written informed consent for the publication of accompanying images in this manuscript. The consent forms are held in the authors' institution and are available for review by the Editor-in-Chief.

\section{Competing interests}

The authors declare that they have no competing interests.

\section{Author details}

${ }^{1}$ Weil Cornell Medicine, Department of Pediatrics, Pediatric Cardiology, 525 East 68th St, F-677, New York, NY 10065, USA. ${ }^{2}$ The Heart Institute, Department of Pediatrics, David's Medical Center, 919 East 32nd Street, Austin, TX 78705, USA. ${ }^{3}$ Department of Radiology, Department of Pediatrics, Cincinnati Children's Hospital Medical Center, 3333 Burnet Ave, Cincinnati, $\mathrm{OH} 45229$, USA. ${ }^{4}$ Division of Biostatistics and Epidemiology, Department of Pediatrics, Cincinnati Children's Hospital Medical Center, 3333 Burnet Ave, Cincinnati, OH 45229, USA. ${ }^{5}$ St. David's Medical Center, 919 East 32nd Street, Austin, TX 78705, USA.

Received: 2 July 2018 Accepted: 14 June 2019

Published online: 08 August 2019

\section{References}

1. Prakash A, Powell AJ, Geva T. Multimodality noninvasive imaging for assessment of congenital heart disease. Circ Cardiovasc Imaging. 2010;3: $112-25$.

2. Babar $J$, Jones RG, Hudsmith L. Application of MR imaging in assessment and follow-up of congenital heart disease in adults. Radiographics. 2010;30: 1-24. https://doi.org/10.1148/rg.e40.

3. Kilner PJ, Geva T, Kaemmerer H, Trindade PT, Schwitter J, Webb GD. Recommendations for cardiovascular magnetic resonance in adults with congenital heart disease from the respective working groups of the European Society of Cardiology. Eur Heart J. 2010;31:794-805.

4. Fratz S, Chung T, Greil GF, Samyn MM, Taylor AM, Valsangiacomo Buechel ER, et al. Guidelines and protocols for cardiovascular magnetic resonance in children and adults with congenital heart disease: SCMR expert consensus group on congenital heart disease. J Cardiovasc Magn Reson. 2013;15:51. https://doi.org/10.1186/1532-429X-15-51.

5. Valsangiacomo Buechel ER, Grosse-Wortmann L, Fratz S, Eichhorn J, Sarikouch S, Greil GF, et al. Indications for cardiovascular magnetic resonance in children with congenital and acquired heart disease: an expert consensus paper of the imaging working group of the AEPC and the cardiovascular magnetic resonance section of the EACVI. Eur Hear J Cardiovasc Imaging. 2015;16:281-97. https://doi.org/10.1093/ehjci/jeu129.

6. Greil G, Tandon A (Aashoo), Silva Vieira M, Hussain T. 3D Whole Heart Imaging for Congenital Heart Disease. Front Pediatr. 2017;5 February:1-10. doi:https://doi.org/10.3389/fped.2017.00036.

7. Greil GF, Powell AJ, Gildein HP, Geva T. Gadolinium-enhanced threedimensional magnetic resonance angiography of pulmonary and systemic venous anomalies. J Am Coll Cardiol. 2002;39:335-41.

8. Geva T, Greil GF, Marshall AC, Landzberg M, Powell AJ. Gadoliniumenhanced 3-dimensional magnetic resonance angiography of pulmonary blood supply in patients with complex pulmonary stenosis or atresia: comparison with x-ray angiography. Circulation. 2002;106:473-8.

9. Valverde I, Parish V, Hussain T, Rosenthal E, Beerbaum P, Krasemann T. Planning of catheter interventions for pulmonary artery stenosis: improved measurement agreement with magnetic resonance angiography using identical angulations. Catheter Cardiovasc Interv. 2011;77:400-8.

10. Sørensen TS, Körperich H, Greil GF, Eichhorn J, Barth P, Meyer H, et al. Operator-independent isotropic three-dimensional magnetic resonance imaging for morphology in congenital heart disease: a validation study. Circulation. 2004;110:163-9.

11. Razavi RS, Hill DLG, Muthurangu V, Miquel ME, Taylor AM, Kozerke S, et al. Three-dimensional magnetic resonance imaging of congenital cardiac anomalies. Cardiol Young. 2003;13:461-5. https://doi.org/10.1017/ S1047951103000957.

12. Bley TA, Wieben $\mathrm{O}$, François $\mathrm{CJ}$, Brittain $\mathrm{JH}$, Reeder SB. Fat and water magnetic resonance imaging. J Magn Reson Imaging. 2010;31:4-18,

13. Su JT, Chung T, Muthupillai R, Pignatelli RH, Kung GC, Diaz LK, et al. Usefulness of real-time navigator magnetic resonance imaging for evaluating coronary artery origins in pediatric patients. Am J Cardiol. 2005; 95:679-82.

14. Beerbaum P, Sarikouch S, Laser KT, Greil G, Burchert W, Körperich H. Coronary anomalies assessed by whole-heart isotropic 3D magnetic resonance imaging for cardiac morphology in congenital heart disease. J Magn Reson Imaging. 2009;29:320-7.

15. Rajiah P, Setser RM, Desai MY, Flamm SD, Arruda JL. Utility of free-breathing, whole-heart, three-dimensional magnetic resonance imaging in the assessment of coronary anatomy for congenital heart disease. Pediatr Cardiol. 2011;32:418-25.

16. Farrelly C, Shah S, Davarpanah A, Keeling AN, Carr JC. ECG-gated multiecho Dixon fat-water separation in cardiac MRI: advantages over conventional fatsaturated imaging. Am J Roentgenol. 2012;199.

17. Dixon WT. Simple proton spectroscopic imaging. Radiology. 1984;153:189-94.

18. Ma J. Dixon techniques for water and fat imaging. J Magn Reson Imaging. 2008;28:543-58.

19. Eggers H, Brendel B, Duijndam A, Herigault G. Dual-echo Dixon imaging with flexible choice of echo times. Magn Reson Med. 2011;65:96-107. https://doi.org/10.1002/mrm.22578.

20. Börnert P, Koken P, Nehrke K, Eggers H, Ostendorf P. Water/fat-resolved whole-heart Dixon coronary MRA: an initial comparison. Magn Reson Med. 2014:71:156-63.

21. Abbara S, Migrino RQ, Sosnovik DE, Leichter JA, Brady TJ. Value of Fat Suppression in the MRI Evaluation of Suspected, March. 2004:587-91.

22. Kellman P, Hernando D, Shah S, Zuehlsdorff S, Jerecic R, Mancini C, et al. Multiecho Dixon fat and water separation method for detecting fibrofatty infiltration in the myocardium. NIH Public Access Methods. 2010;61:215-21.

23. Homsi R, Sprinkart AM, Gieseke J, Yuecel S, Meier-Schroers M, Luetkens J, et al. 3D-Dixon cardiac magnetic resonance detects an increased epicardial fat volume in hypertensive men with myocardial infarction. Eur J Radiol. 2016;85:936-42.

24. Leiner T, Habets J, Versluis B, Geerts L, Alberts E, Blanken N, et al. Subtractionless first-pass single contrast medium dose peripheral MR angiography using twopoint Dixon fat suppression. Eur Radiol. 2013;23:2228-35.

25. Homsi R, Gieseke J, Kukuk GM, Träber F, Willinek WA, Schild HH, et al. Dixonbased fat-free MR-angiography compared to first pass and steady-state high-resolution MR-angiography using a blood pool contrast agent. Magn Reson Imaging. 2015;33:1035-42. https://doi.org/10.1016/j.mri.2015.07.005.

26. Molière S, Dillenseger JP, Ehlinger M, Kremer S, Bierry G. Comparative study of fatsuppression techniques for hip arthroplasty MR imaging. Skelet Radiol. 2017:46: 1209-17.

27. Greil GF, Powell AJ, Gildein HP, Geva T. Gadolinium-enhanced threedimensional magnetic resonance angiography of pulmonary and systemic venous anomalies. J Am Coll Cardiol. 2002;39:335-41. https://doi.org/10. 1016/S0735-1097(01)01730-2.

28. Valsangiacomo Büchel ER, DiBernardo S, Bauersfeld U, Berger F. Contrastenhanced magnetic resonance angiography of the great arteries in patients with congenital heart disease: an accurate tool for planning catheter-guided interventions. Int J Cardiovasc Imaging. 2005;21:313-22. https://doi.org/10. 1007/s10554-004-4017-y.

29. Kawel-Boehm N, Maceira A, Valsangiacomo-Buechel ER, Vogel-Claussen J, Turkbey EB, Williams R, et al. Normal values for cardiovascular magnetic resonance in adults and children. J Cardiovasc Magn Reson. 2015;17:1-33.

30. Stinson EG, Trzasko JD, Weavers PT, Riederer SJ. Dixon-type and subtractiontype contrast-enhanced magnetic resonance angiography: a theoretical and experimental comparison of SNR and CNR. Magn Reson Med. 2015;74:81-92.

31. Riederer SJ, Stinson EG, Weavers PT. Technical aspects of contrast-enhanced MR angiography: current status and new applications. Magn Reson Med Sci. 2017:1-10. https://doi.org/10.2463/mrms.rev.2017-0053.

32. Hernando D, Kellman P, Haldar JP, Liang ZP. Robust water/fat separation in the presence of large field inhomogeneities using a graph cut algorithm. Magn Reson Med. 2010;63:79-90. 
33. Nezafat M, Henningsson M, Ripley DP, Dedieu N, Greil G, Greenwood JP, et al. Coronary MR angiography at 3T: fat suppression versus water-fat separation. Magn Reson Mater Physics, Biol Med. 2016;29:733-8.

34. Le Y, Kipfer HD, Majidi SS, Holz S, Lin C. Comparison of the artifacts caused by metallic implants in breast MRI using dual-echo Dixon versus conventional fat-suppression techniques. Am J Roentgenol. 2014;202: W307-14.

35. Feltes TF, Bacha E, Beekman RH, Cheatham JP, Feinstein JA, Gomes AS, et al. Indications for cardiac catheterization and intervention in pediatric cardiac disease: a scientific statement from the American Heart Association. Circulation. 2011;123:2607-52.

36. Pool B, Makowski MR, Wiethoff AJ, Uribe S, Bell A, Kiesewetter C, et al. Congenital heart disease: cardiovascular MR imaging by using an intravascular blood pool contrast agent. Radiology. 2011;260:680-8. https:// doi.org/10.1148/radiol.11102327.

37. Cheng L, Gao Y, Guaricci Al, Mulukutla S, Sun W, Sheng F, et al. Breath-hold 3D steady-state free precession coronary MRA compared with conventional x-ray coronary angiography. J Magn Reson Imaging. 2006;23:669-73.

38. Michaely HJ, Attenberger UI, Dietrich O, Schmitt P, Nael K, Kramer H, Reiser MF, Schoenberg SO, Walz M. Feasibility of gadofosveset-enhanced steadystate magnetic resonance angiography of the peripheral vessels at 3Tesla with Dixon fat saturation. Investig Radiol. 2008;43:635-41.

39. Stinson EG, Trzasko JD, Campeau NG, et al. Time-resolved contrastenhanced MR angiography with single-echo Dixon fat suppression. Magn Reson Med. 2018;00:1-12.

\section{Publisher's Note}

Springer Nature remains neutral with regard to jurisdictional claims in published maps and institutional affiliations.

Ready to submit your research? Choose BMC and benefit from:

- fast, convenient online submission

- thorough peer review by experienced researchers in your field

- rapid publication on acceptance

- support for research data, including large and complex data types

- gold Open Access which fosters wider collaboration and increased citations

- maximum visibility for your research: over $100 \mathrm{M}$ website views per year

At $\mathrm{BMC}$, research is always in progress.

Learn more biomedcentral.com/submissions 